\title{
EDITORIAL
}

\section{Integrated care: a new model for COPD management?}

\author{
T.A.R. Seemungal* and J.A. Wedzicha ${ }^{\#}$
}

$\mathbf{T}$ otal healthcare expenditure in countries of the Organisation for Economic Co-operation and Development has risen from an average of $5 \%$ of gross domestic product in 1970 to $\sim 10 \%$ in 2002 [1]. In 2002, pharmaceutical expenditure ranged from $10 \%$ of the total healthcare expenditure in Sweden to $21 \%$ in France [1], and a major portion of this expenditure arose through hospital care. Chronic obstructive pulmonary disease (COPD) is one of the most common chronic diseases worldwide [2] and a common cause of hospitalisation. An analysis of the economic cost of COPD in the UK shows that $54 \%$ accrues from hospitalisation; a further $32 \%$ is equally divided between scheduled care and drug treatment [3]. The Study of Risk Factors of COPD Exacerbation (EFRAM Study) showed that among a wide range of potential risk factors, only previous admissions, lower forced expiratory volume in one second, and the underprescription of oxygen are independently associated with a higher risk of admission for a COPD exacerbation [4]. Underprescription of home oxygen is easily modifiable once hypoxia has been diagnosed, but hospital admission rates for COPD remain a challenge for pulmonologists and chest therapists alike. In the current issue of the European Respiratory Journal, there is a new and interesting attempt at management of the severe COPD patient. CASAS et al. [5] advance a model that may have great potential for the cross-cultural modification of hospitalisation rates for COPD. Previous studies have shown that self-management of COPD within the community can reduce hospitalisation [6] and exacerbation severity [6, 7]. The economic efficacy of these models is unknown, but since hospitalisation contributes the greatest proportion of costs for COPD, models targeting patients at risk for hospitalisation may be expected to have the widest appeal to chest physicians and healthcare providers alike.

Several attempts have been made at establishing interventions for patients with chronic illnesses, almost all of which include prevention of hospitalisation as an outcome measure and almost always within one cultural environment. A metaanalysis of 102 such studies has shown that commonly used interventions have included patient education, healthcare provider education, and provider feedback. All such studies were associated with improvements in disease control and

\footnotetext{
*Dept of Clinical Medical Sciences, University of West Indies, St Augustine Campus, Trinidad and Tobago. ${ }^{\#}$ Royal Free and University College Medical School, University College London, London, UK.

CORRESPONDENCE: T.A.R. Seemungal, Dept of Clinical Medical Sciences, Faculty of Medical Science, University of West Indies, St Augustine Campus, Trinidad and Tobago. Fax: 8686634332. E-mail: tseemungal@aol.com
}

adherence to practice guidelines, but because of the diversity of outcome measures and structure, little else could be concluded [8]. In COPD, such studies have recruited patients from the community or hospital. The East London COPD study, a long-term cohort study of moderate-to-severe COPD patients in the community, showed that over a period of 6 yrs hospitalisation rates were higher amongst those COPD patients who tended not to seek treatment for an exacerbation compared with those who sought early treatment from family physicians or the study clinicians [7]. BOURBEAU et al. [9] studied 191 COPD patients of similar severity recruited from the outpatient clinics of seven Canadian hospitals, randomised to a self-management plan or usual care (UC), and showed that self-managed subjects were less likely to be hospitalised during the first and also the second year, when there was less contact with the study team [6]. While the East London study looked at unselected patients in the community, the study by BOURBEAU et al. [9] required prior hospitalisation in the previous year as a prerequisite $[7,9]$. However, a randomised study of self-management versus UC involving 248 COPD community-based patients from the Netherlands showed no measurable beneficial effects of self-management on qualityadjusted life years, or health-related quality of life, but this study did not require prior hospitalisation as a prerequisite and the patients were of milder severity than in either of the previous two studies [10]. This suggests that perhaps selfmanagement programmes should focus on the more severe patients who tend to use most resources.

In order to decrease the cost of hospitalised patients, supported discharge or hospitals at home schemes have been advocated. Where generic management home-based schemes have been used for chronic disease patients, no benefit has been found for COPD [11-14]. A Scottish observational study of 962 patients with COPD also found benefit for hospital-led home-based care [15], but a recent Australian randomised controlled study found no benefit [16]. However, the number of randomised controlled trials using this approach has been relatively small. In a systematic review, RAM et al. [17] found seven such trials involving a total of only 754 patients, but were able to conclude that home-based schemes are associated with cost savings as well as decreased hospitalisation. All of these studies have used differing models, but commonly involve a respiratory therapist or senior nurse case manager. Furthermore, in a systematic review of nine randomised controlled trials of inpatient, outpatient and community-based interventions (excluding hospital at home and early discharge schemes), TAYLOR et al. [18] have cautioned that even though the data are sparse, there is currently little evidence to support purely nurse-led management interventions for COPD. 
CASAS et al. [5] utilised an innovative approach for this problem, and several features of this study are of interest. First, the authors studied hospitalised COPD patients in two different cultural environments, Belgium and Spain. Secondly, apart from including a specialised nurse case manager, they incorporated a chronic platform, which was a web-based application that facilitated phone calls to the case manager, as well as record keeping via phone calls, home visits or home video conferencing. In a study of 217 adults with type-2 diabetes, GLASGOW et al. [19] concluded that physicians and patients were willing to participate in computer-assisted diabetes self-management and that results appeared robust over various patient and delivery characteristics. A small study from Athens (Greece) has also suggested that telemedicine home support of severe COPD patients may decrease hospitalisations [20]. The use of computer-assisted care for chronic diseases has been slow in coming, but will receive greater attention during this decade and the authors should be commended for having the foresight to use this increasingly available resource in their methodology.

Unlike hospital-led home-based studies, the hospital stay of patients in the study by CASAS et al. [5] was not truncated by access to the study as patients were recruited at discharge determined by local protocols. However, at discharge, there was careful evaluation for access into the study as well as a 2-h educational session. The case management was customised to location. Thus, in Belgium, general practitioners (GPs) were included via regional education sessions and were specifically contacted by one of the researchers; however, in Spain, there was one joint visit involving the case manager and the primary care team within $72 \mathrm{~h}$ of discharge. Phone calls were made weekly for 1 month, then at 3 and 9 months. The study showed that this integrated care (IC) model is effective in preventing hospital admissions with specifics adapted to differing local health environments. The study did not include evaluation of GP training in Belgium where there was a greater emphasis on GP involvement. However, the frequency of GP visits did not differ between the UC or IC arms, and, since the IC arm was associated with better outcomes, this may be taken as indirect positive evaluation of GP training in the Belgium arm of the study.

Some limitations, as well as implications, of the study should be mentioned. Deaths in both the UC and IC arms were similar, with the majority being from end-stage pulmonary disease. However, there was no evidence of differences in death rates from exacerbations of COPD in either arm, although this was not an outcome measure of this study and should be a matter for careful evaluation in further studies. CASAs et al. [5] have explained in some detail why the randomisation process was altered in one of the centres, but this does not appear to have biased the results. With the fall off in frequency of programmed phone calls with time, the intensity of case manager involvement would be expected to decline. The effect of this aspect of the study design on hospitalisation rates was not assessed during the study, but it should be noted that hospitalisation rates in the second year when contact times had fallen were unaffected in the Canadian study alluded to earlier [6]. Interestingly there were no home visits of the case manager in Belgium due to the geographical dispersion of the patients, but home visits were part of the protocol in Spain, yet the hazard ratio for hospitalisation at Belgium was 0.35, compared with that of 0.52 in Spain. Thus, one of the inferences from this study is that where there is good communication with primary care, there may be no need for home visits from secondary or tertiary care facilities.

One further important question that all healthcare providers will want to ask is which patients should be enrolled into these programmes? The major problem with community-recruited self-management models is that they have a recruitment rate of only $50 \%[6,9]$, whereas supported discharge and IC models originating within hospitals have a much higher recruitment rate: CASAS et al. [5] had a refusal of only 3\%. One apparently disappointing feature of the study is that only $19 \%$ of admitted patients met the stringent inclusion criteria. The study required home visits and thus the $39 \%$ of patients not in the catchment area had to be excluded and, quite reasonably, a further $10 \%$ who were illiterate could not be included, as they had to be able to make use of the chronic platform. A further $32 \%$ with severe comorbidity who probably required a separate IC pathway were also excluded. Obviously, the exclusion of $39 \%$ of patients because of the catchment area can represent a potential source of bias, but we may be reassured that these patients differed in being only slightly more likely to be females and less likely to be smokers than those included; importantly, they did not differ in severity as measured by the need for intensive care. Thus, the integrated pathway of care is available for all COPD patients, without significant comorbidity, provided they are literate.

The main message of the study is that an integrated care pathway with flexible shared-care arrangements between primary care and hospital, facilitated by information technologies, has an enormous potential to decrease hospital admissions in chronic obstructive pulmonary disease patients. In the integrated care approach, several models are available and we now require comparative studies. However, we must also be aware that from a healthcare provider perspective, further studies of this model must include cost-effectiveness as well as effectiveness in older disabled patients with chronic obstructive pulmonary disease.

\section{REFERENCES}

1 Yuen P. OHE Compendium 2004-2005. 16th Edn. Oxford, Radcliffe Publishing, 2005.

2 Murray CJL, Lopez AD. Alternative projections of mortality and disability by cause 1990-2020: Global Burden of Disease Study. Lancet 1997; 349: 1498-1504.

3 Britton M. The burden of COPD in the U.K.: results from the Confronting COPD survey. Respir Med 2003; 97: Suppl. C, S71-S79.

4 Garcia-Aymerich J, Monso E, Marrades RM, et al. Risk factors for hospitalization for a chronic obstructive pulmonary disease exacerbation. EFRAM study. Am J Respir Crit Care Med 2001; 164: 1002-1007.

5 Casas A, Troosters T, Garcia-Aymerich J, et al. Integrated care prevents hospitalisations for exacerbations in COPD patients. Eur Respir J 2006; 28: 123-130.

6 Gadoury M-A, Schwartzman K, Rouleau M, et al. Selfmanagement reduces both short- and long-term hospitalisation in COPD. Eur Respir J 2005; 26: 853-857. 
7 Wilkinson TMA, Donaldson GC, Hurst JR, Seemungal TAR, Wedzicha JA. Early therapy improves outcomes of exacerbations of chronic obstructive pulmonary disease. Am J Respir Crit Care Med 2004; 169: 1298-1303.

8 Weingarten SR, Henning JM, Badamgarav E, et al. Interventions used in disease management programmes for patients with chronic illness-which ones work? Metaanalysis of published reports. BMJ 2002; 325: 925.

9 Bourbeau J, Julien M, Maltais F, et al. Reduction of hospital utilization in patients with chronic obstructive pulmonary disease: a disease specific self-management intervention. Arch Intern Med 2003; 163: 585-591.

10 Monninkhof E, van der Valk P, Schermer T, van der Palen J, van Herwaarden C, Zielhuis G. Economic evaluation of a comprehensive self-management programme in patients with moderate to severe chronic obstructive disease. Chron Respir Dis 2004; 1: 7-16.

11 Pearson S, Inglis SC, McLennan SN, et al. Prolonged effects of a home-based intervention in patients with chronic illness. Arch Intern Med 2006; 166: 645-650.

12 Kwok T, Lum CM, Chan HS, Ma HM, Lee D, Woo J. A randomized, controlled trial of an intensive community nurse-supported discharge program in preventing hospital readmissions of older patients with chronic lung disease. J Am Geriatr Soc 2004; 52: 1240-1246.

13 Shepperd S, Harwood D, Gray A, et al. Randomised controlled trial comparing hospital at home care with inpatient hospital care. II: cost minimisation analysis. BMJ 1998; 316: 1791-1796.
14 Shepperd S, Harwood D, Jenkinson C, Gray A, Vessey M, Morgan P. Randomised controlled trial comparing hospital at home care with inpatient hospital care. I: three month follow up of health outcomes. BMJ 1998; 316: 1786-1791.

15 Gravil JH, Al-Rawas OA, Cotton MM, Flanigan U, Irwin A, Stevenson RD. Home treatment of exacerbations of chronic obstructive pulmonary disease by an acute respiratory assessment service. Lancet 1998; 351: 1853-1855.

16 Hermiz O, Comino E, Marks G, Daffurn K, Wilson S, Harris M. Randomised controlled trial of home based care of patients with chronic obstructive pulmonary disease. BMJ 2002; 325: 938.

17 Ram FSF, Wedzicha JA, Wright J, Greenstone M. Hospital at home for patients with acute exacerbations of chronic obstructive pulmonary disease: systematic review of evidence. BMJ 2004; 329: 315.

18 Taylor SJC, Candy B, Bryar RM, et al. Effectiveness of innovations in nurse led chronic disease management for patients with chronic obstructive pulmonary disease: systematic review of evidence. BMJ 2005; 331: 485.

19 Glasgow RE, Strycker L, King DK, et al. Robustness of a computer assisted diabetes self management intervention across characteristics, healthcare settings and intervention staff. Am J Manag Care 2006; 12: 137-145.

20 Vontetsianos T, Giovas P, Katsaras T, et al. Telemedicineassisted home support for patients with advanced chronic obstructive pulmonary disease: preliminary results after nine-month follow-up. J Telemed Telcare 2005; 11: Suppl. 1, 86-88. 